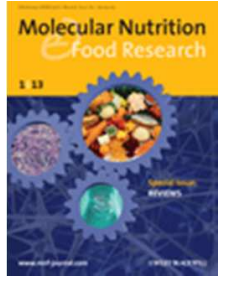

\title{
A low-protein diet induces body weight loss and browning of subcutaneous white adipose tissue through enhanced expression of hepatic Fibroblast Growth Factor 21 (FGF21).
}

\begin{tabular}{|r|l|}
\hline Journal: & Molecular Nutrition and Food Research \\
\hline Manuscript ID & mnfr.201600725 \\
\hline Datey - Manuscript type: & Research Article \\
\hline Complete List of Authors: & $\begin{array}{l}\text { Pérez-Martí, Albert; University of Barcelona, Nutrition, Food Sciences and } \\
\text { Gastronomy. School of Pharmacy and Food Science } \\
\text { Garcia-Guasch, Maite; University of Barcelona, Nutrition, Food Sciences } \\
\text { and Gastronomy. School of Pharmacy and Food Science } \\
\text { Tresserra-Rimbau, Anna; University of Barcelona, Nutrition, Food Sciences } \\
\text { and Gastronomy. School of Pharmacy and Food Science } \\
\text { Estruch, Ramon; University of Barcelona, Department of Internal Medicine, } \\
\text { Hospital Clinic, IDIBAPS } \\
\text { Salas-Salvado, J; Rovira i Virgili University } \\
\text { Martínez-González, Miguel Ángel; Universidad de Navarra, Medicina } \\
\text { Preventiva y Salud Pública } \\
\text { Lamuela Raventos, Rosa; University of Barcelona, Department of Nutrition } \\
\text { and Food Science. School of Pharmacy and Food Science } \\
\text { Marrero, Pedro; University of Barcelona, Nutrition, Food Sciences and } \\
\text { Gastronomy. School of Pharmacy and Food Science } \\
\text { Haro, Diego; University of Barcelona, Nutrition, Food Sciences and } \\
\text { Gastronomy. School of Pharmacy and Food Science } \\
\text { Relat, Joana; University of Barcelona, Nutrition, Food Sciences and } \\
\text { Gastronomy. School of Pharmacy and Food Science }\end{array}$ \\
\hline Keywords: & $\begin{array}{l}\text { Adipose tissue, Browning, Fibroblast growth factor 21, Low-protein diet, } \\
\text { Uncoupling protein 1 }\end{array}$ \\
\hline & \begin{tabular}{l}
$\mid$ \\
\hline
\end{tabular} \\
\hline
\end{tabular}


A low-protein diet induces body weight loss and browning of subcutaneous white adipose tissue through enhanced expression of hepatic Fibroblast Growth Factor 21 (FGF21).

Albert Pérez-Martí ${ }^{1,2}$, Maite Garcia-Guasch ${ }^{1,2}$, Anna Tresserra-Rimbau ${ }^{1,3,4}$, Ramon Estruch $^{4,5}$, Jordi Salas-Salvadó ${ }^{4,6}$, Miguel Ángel Martínez-González ${ }^{4,7}$, Rosa LamuelaRaventós ${ }^{1,3,4}$, Pedro F. Marrero ${ }^{1,2}$, Diego Haro ${ }^{1,2 *}$, Joana Relat ${ }^{1,2 *}$.

${ }^{1}$ Department of Nutrition, Food Sciences and Gastronomy, School of Pharmacy and Food Science, University of Barcelona, Torribera Food Campus, Santa Coloma de Gramenet, (Barcelona) Spain.

${ }^{2}$ Institute of Biomedicine of the University of Barcelona (IBUB).

${ }^{3}$ Insitute of Nutrition and Food Safety of the University of Barcelona (INSA-UB).

${ }^{4}$ CIBEROBN, Instituto de Salud Carlos III, Madrid, Spain.

${ }^{5}$ Department of Internal Medicine, Hospital Clinic, IDIBAPS, University of Barcelona, Spain.

${ }^{6}$ Human Nutrition Department, Hospital Universitari Sant Joan, Institut d'Investigació Sanitària Pere Virgili, University Rovira i Virgili, Reus (Tarragona), Spain.

${ }^{7}$ Department of Preventive Medicine and Public Health, Universidad de NavarraInstitute of Health Research of Navarra (IDISNA), Pamplona, Spain.

*Corresponding Authors: Diego Haro (dharo@ub.edu) and Joana Relat (jrelat@ub.edu)

${ }^{1}$ Department of Nutrition, Food Sciences and Gastronomy, School of Pharmacy, University of Barcelona, Torribera Food Campus, Santa Coloma de Gramenet, (Barcelona) Spain. Phone: +34934020862 Fax: +34934024520 .

\begin{abstract}
Abbreviations: Fibroblast growth factor 21 (FGF21), Low-protein diet (LPD), white adipose tissue (WAT), Uncoupling protein 1 (UCP1), Energy expenditure (EE), Type 2 iodothyronine deiodinase protein 2 (DIO2), Brown adipose tissue (BAT), Peroxisome proliferator-activated receptor (PPAR), Fatty acid oxidation (FAO), Activating transcription factor 4 (ATF4), Diet-induced obesity (DIO), High fat diet (HFD), Control
\end{abstract}


diet (CD), Quantitative food frequency questionnaire (FFQ), Peroxisome proliferatoractivated receptor gamma coactivator 1-alpha (PGC1a), PR domain containing 16 (PRDM16).

Keywords: Adipose tissue; browning; Fibroblast growth factor 21; low-protein diet; Uncoupling protein 1. 


\section{ABSTRACT}

Scope: Fibroblast growth factor 21 (FGF21) is considered a promising therapeutic candidate for the treatment of obesity. Since FGF21 production is regulated by various nutritional factors, we analyze the impact of low protein intake on circulating levels of this growth hormone in mice and in a sub cohort of the PREDIMED (Prevención con Dieta Mediterránea) trial. We also describe the role of hepatic FGF21 in metabolic adaptation to a low-protein diet (LPD).

Methods and results: We fed control and liver-specific Fgf21 knockout (LFgf21KO) mice a LPD. This diet increased FGF21 production by inducing its overexpression in liver, and this correlated with a body weight decrease without changes in food intake. The LPD also caused FGF21-dependent browning in subcutaneous white adipose tissue (scWAT), as indicated by an increase in the expression of uncoupling protein 1 (UCP1). In a subgroup of 78 individuals from the PREDIMED trial, we observed an inverse correlation between protein intake and circulating FGF21 levels.

Conclusions: Our results reinforce the involvement of FGF21 in coordinating energy homeostasis under a range of nutritional conditions. Moreover, here we describe an approach to increase the endogenous production of FGF21 in humans that may pave the way for a treatment for obesity. 


\section{INTRODUCTION}

FGF21 (Fibroblast growth factor 21) is considered a promising therapeutic candidate for the treatment of obesity and type-2 diabetes. Its administration to obese rodents and monkeys leads to decreased plasma concentrations of glucose, insulin, triglycerides and cholesterol, as well as a reduction in body weight through increased energy expenditure (EE) [1]. The injection of FGF21 in experimental animals induces increased thermogenic capacity by stimulating the expression of uncoupling protein 1 (UCP1) and type 2 iodothyronine deiodinase protein 2 (DIO2) in brown adipose tissue (BAT), and UCP1 in white adipose tissue (WAT), where it produces the so-called browning process [2,3]. Although most of the effects of FGF21 have been related to UCP1 expression, it has also been reported that Ucp1-null mice respond positively to the pharmacological administration of this growth factor $[4,5]$.

FGF21 is a member of the FGF family, which is characterized by endocrine properties. It is produced mainly by the liver, but also by other tissues such as WAT, BAT, skeletal muscle, and pancreas [6-9]. Hepatic FGF21 expression is induced by fasting, and its transcriptional activity is tightly controlled by peroxisome proliferator-activated receptor alpha (PPARa) [10-13]. The expression of this growth factor in liver activates fatty acid oxidation (FAO), ketogenesis and gluconeogenesis in this organ, thereby triggering a metabolic state that mimics long-term fasting [14].

In addition to fasting, FGF21 expression is also induced in various tissues in response to a number of nutritional challenges and also to cold exposure. In this regard, FGF21 expression in BAT is produced in response to cold temperature $[9,15]$, although it is unclear whether BAT-derived FGF21 acts as an endocrine factor or whether it is simply an autocrine factor in the adipose tissue itself.

In mouse liver and HepG2 cells, FGF21 is induced by leucine-deprivation as part of the transcriptional program initiated by increased levels of activating transcription factor 4 (ATF4) [16]. The ATF4-dependent increase in FGF21 expression has been confirmed 
in mice with autophagy deficiency in skeletal muscle and in liver [17]. Interestingly, these mice are protected from diet-induced obesity (DIO) and insulin resistance. The similarities in the metabolic responses between the effects to leucine-deprivation [18] and to FGF21 overexpression allowed us to consider FGF21 as a key mediator between amino acid deprivation and lipid metabolism in liver, WAT and BAT. In this regard, results from the evaluation of the metabolic response of Fgf21-deficient mice to a leucine-deficient diet previously led us to conclude that, as expected, most of the effects caused by leucine deprivation in liver, WAT and BAT are impaired in the absence of this growth factor [19].

Likewise, methionine-deprived mice show a phenotype comparable to that of leucinedeprivation, including resistance to a high-fat diet (HFD), improved glucose homeostasis, increased fatty acid activation and FAO in liver, enhanced lipolysis in WAT, and increased UCP1 expression in BAT $[20,21]$. Of note, the induction of hepatic FGF21 expression under leucine- or methionine-restricted diets was found to be accompanied by an increase in FGF21 protein levels in serum.

In order to facilitate the translation of these findings to humans, here we focussed on low-protein diets (LPD) instead of amino acid-deficient diets. Protein restriction brings about weight loss and an increase in both food intake and EE [22]. Moreover, a LPD induces thermogenic markers in BAT of obese rats [23]. Moreover, serum concentrations of FGF21 in both rodents and humans increase upon exposure to a LPD, regardless of total calorie intake. This observation thus reveals that FGF21 is likely to be involved in the metabolic response to protein-restricted diets [24].

Here we addressed whether a LPD exerts similar effects on lipid metabolism to those of a leucine-deficient diet and whether these effects are dependent on hepatic FGF21 production. To this end, we examined the metabolic response of wild-type and Fgf21 liver-specific knockout mice (LFgf21KO) to a LPD (up to $5 \%$ of energy as protein). A decreased in dietary protein content induced a huge increase in FGF21 serum levels, 
significant weight loss, and an increase in the expression of UCP1 in the subcutaneous WAT (scWAT) of wild-type mice. Remarkably, no effects were observed in Fgf21deficient mice, thereby indicating that the absence of FGF21 blunts or completely blocks the response to a LPD in this mouse model .

To corroborate these results in humans, we evaluated whether protein intake is associated with circulating levels of FGF21. We calculated protein intake through nutritional questionnaires and determined the serum levels of FGF21 in 78 individuals randomly selected from two nodes of the PREDIMED (Prevención con Dieta Mediterránea) trial. As with the animal model, an inverse correlation between circulating FGF21 levels and protein intake was observed.

To summarize, here we define the molecular mechanisms by which a LPD exerts its metabolic effects through the induction of hepatic FGF21 expression and browning of scWAT. Furthermore, the data collected from humans raises the possibility of an alternative approach to modulate the FGF21 circulating levels instead of its pharmacological administration. In this regard, we propose the modification of protein intake to enhance FGF21 production.

\section{MATERIAL AND METHODS}

\section{Animals.}

To generate the LFgf21KO mice, Fgf21 ${ }^{\text {loxP }}$ mice $\left(F g f 21^{\text {tm1.2Djm } / J)}\right.$ that have Fgf21 flanked by two loxP sites (Jackson Laboratory, USA) were crossed with Albumin-cre (Tg(Alb1-cre)1Dlr/J) mice (kindly provided by Dr. A. Zorzano). The latter express the CRE recombinase enzyme under control of albumin promoter/enhancer elements, thus allowing liver-specific gene deletions [25]. Fgf2 ${ }^{\text {LoxP }}$ mice were used as controls. Animals were housed in a temperature-controlled room $\left(22 \pm 1^{\circ} \mathrm{C}\right)$ on a $12 / 12 \mathrm{~h}$ light/dark cycle and were provided free access to commercial rodent chow and tap water prior to the experiments. 


\section{Dietary intervention.}

The control diet (CD) (Ref. D10001) and LPD (Ref. D12010401) were obtained from Research Diets, Inc. (USA). Both diets were isocaloric. They had the following composition (in percentage of mass): $20 \%$ protein, $66 \%$ carbohydrates and $5 \%$ fat for the $C D$, and $5 \%$ protein, $81 \%$ carbohydrates and $5 \%$ fat for the LPD (detailed composition shown in Table S1). For the feeding experiment, 8-week-old male mice were first fed the CD for 7 days and then randomly assigned to either the CD or LPD group with free access to food and water for 7 days. Food intake and body weight were recorded daily. Animals were then anesthetized by isoflurane inhalation, and blood was collected by cardiac puncture. After euthanizing the animals, tissues were isolated and immediately snap-frozen and stored at $-80^{\circ} \mathrm{C}$ for future analysis. The Animal Ethics Committee of the University of Barcelona approved these experiments (CEEA register: 48/15)

\section{Human samples for plasma measurements.}

We used plasma samples from 78 men and women randomly selected from the participants of the centers in Hospital Clinic (Barcelona) and Reus (Tarragona) of the PREDIMED trial (www.predimed.es). This study was a 5-year randomized clinical trial to compare the effects of either a Mediterranean diet supplemented with extra virgin olive oil or nuts versus a low-fat control diet. A total of 7447 asymptomatic men but at high cardiovascular risk (aged 55-80 years) and women (aged 60-80 years) were recruited. All participants had type 2 diabetes or three or more cardiovascular risk factors. Details of the recruitment method and study design have been described elsewhere [26] and are also available at www.predimed.es. In addition to the plasma samples, we also gathered information from these 78 individuals, including a 137 -item semi-quantitative food frequency questionnaire (FFQ), and a general questionnaire that provided data on lifestyle habits, concurrent diseases, anthropometry, and medication use. Total energy intake and nutrient intake were calculated on the basis of Spanish 
food composition tables [27]. The study protocol was approved by the institutional review boards of the participating centers (ISRCTN35739639).

\section{Plasma measurements.}

Mouse plasma samples were obtained by centrifuging whole blood collected in EDTAtreated tube. The plasma was stored at $-80^{\circ} \mathrm{C}$. FGF21 in mouse and human plasma was measured by means of a Human (ref. EZHFGF21-19K) and Mouse/Rat (ref. EZRMFGF21-26K) FGF21 ELISA obtained from EMD Millipore (Germany). The assay was conducted following the manufacturer's protocol. Briefly, a calibration curve was constructed by plotting the difference in absorbance values at 450 and $590 \mathrm{~nm}$ versus the FGF21 concentrations of the calibrators, and concentrations of unknown samples (performed in duplicate) were determined using this calibration curve.

\section{RNA isolation and relative quantitative $R T-P C R$}

Total RNA was extracted from the frozen tissues [liver, epididymal WAT (eWAT), BAT and inguinal scWAT] using TRI reagent solution (ref. AM9738 Ambion, Thermo Fisher Scientific, USA) followed by DNase I treatment (ref. AM1906, Ambion, Thermo Fisher Scientific, USA) to eliminate genomic DNA contamination. To measure the relative mRNA levels, quantitative (q)RT-PCR was performed using SYBR Green or TaqMan reagents. cDNA was synthesized from $1 \mu \mathrm{g}$ of total RNA by MLV reverse transcriptase (ref. 28025021, Invitrogen, Thermo Fisher Scientific, USA) with random hexamers (ref. 11034731001, Roche Diagnostics, Germany), following the manufacturer's instructions. The TaqMan Gene Expression Master Mix (ref. 4369514) and SYBR® Green PCR Master Mix (ref. 4364344), supplied by Applied Biosystems (ThermoFisher Scientific, USA), were used for the PCR step. Amplification and detection were performed using the Step-One Plus Real-Time PCR System (Applied Biosystems, ThermoFisher Scientific, USA). Each mRNA from a single sample was measured in duplicate, using 18S, Beta-Actin, and $36 \mathrm{~b} 4$ as housekeeping genes. The primer sequences are shown 
in supplemental information Table S2. Results were obtained by the Relative Standard Curve Method and expressed as fold increase versus the experimental control.

\section{Protein extracts preparation.}

To obtain liver nuclear extracts, frozen liver was triturated with a mortar in liquid nitrogen and immediately homogenized with a Dounce in $1 \mathrm{ml}$ of HB buffer [15 mM Tris- $\mathrm{HCl}(\mathrm{pH}$ 8), $15 \mathrm{mM} \mathrm{NaCl}, 60 \mathrm{mM} \mathrm{KCl}, 0.5 \mathrm{mM}$ EDTA], and centrifuged at $800 \mathrm{~g}$ for $5 \mathrm{~min}$. The resulting pellet was resuspended in $100 \mu \mathrm{l}$ of HB buffer supplemented with $0.05 \%$ Triton X-100 (Sigma, USA) and centrifuged for $10 \mathrm{~min}$ at $1,000 \mathrm{~g}$. Nuclear pellets were washed with $1 \mathrm{ml}$ of HB buffer supplemented with $0.05 \%$ Triton X-100 and $1 \mathrm{ml}$ of HB buffer. Nuclei were incubated at $4^{\circ} \mathrm{C}$ for $30 \mathrm{~min}$ in $50 \mu \mathrm{l}$ of HB buffer containing $360 \mathrm{mM}$ of $\mathrm{KCl}$ and then centrifuged for $5 \mathrm{~min}$ at $10,000 \mathrm{~g}$. The supernatants corresponding to the nuclear extracts were collected, frozen, and stored at $-80^{\circ} \mathrm{C}$. Protein concentration was determined using the Bio-Rad Protein Assay Dye Reagent Concentrate (Ref. 5000006, Bio Rad, USA). All buffers were supplemented with a mixture of protease inhibitors (Ref. P8340, Sigma-Aldrich, USA), $0.1 \mathrm{mM}$ of PMSF, and a phosphatase inhibitor cocktail 3 (Ref. P0044, Sigma-Aldrich, USA).

\section{Immunoblotting.}

Nuclear proteins were resolved by SDS-polyacrylamide gel electrophoresis and transferred onto a Hybond-P PVDF membrane (Millipore). Membranes were blocked (Tris- $\mathrm{HCl} 50 \mathrm{mM} \mathrm{pH} \mathrm{8,} 150 \mathrm{mM}, 5 \%$ skimmed milk, $0.1 \%$ Tween) for $1 \mathrm{~h}$ at room temperature. The blots were then incubated with ATF4 primary antibody (sc-200, Santa Cruz Biotechnology, Inc, USA) in blocking solution (1:200). After an overnight incubation at $4^{\circ} \mathrm{C}$, the blots were washed and incubated with an anti-rabbit horseradish peroxidase-conjugated secondary antibody (ref. NA934, Amersham, GE Healthcare, UK) in blocking buffer for $2 \mathrm{~h}$ at room temperature. The blots were developed using the EZ-ECL Chemiluminescence Detection Kit for HRP (ref. 20-500-500, Biological Industries, Israel). Quantification was performed using Image J software. 


\section{Data analysis / statistics.}

For human samples, baseline characteristics are presented as means \pm standard error of the mean (SEM) for continuous variables, frequencies and percentages for categorical variables across quartiles of protein intake at baseline. Differences between quartiles were tested by a 1-factor ANOVA test for continuous variables and by the chisquare test for the categorical ones. We performed multiple linear regressions to evaluate the relationship between protein intake (exposure variable) and FGF21 hormone levels (dependent variable). Protein intake was previously adjusted for calories using the residual method. Regression analyses were unadjusted (model 1) or adjusted by body mass index (BMI) and total energy intake (model 2)

All statistical analyses were conducted using SAS software, version 9.3 (SAS Institute, Inc., USA). All $t$ tests were 2 -sided and $P$ values below 0.05 were considered statistically significant.

\section{RESULTS AND DISCUSSION}

\section{A LPD induces FGF21 gene expression in the liver of control mice, but not in BAT or WAT.}

According to previously reported results, mice on a LPD show a dramatic increase in serum levels of FGF21 [22-24] (Figure 1A). To check the origin of this FGF21, we analyzed the Fgf21 mRNA levels in several tissues. The liver is the main site of FGF21 production and release into the blood. Accordingly, we observed a great induction of Fgf21 mRNA synthesis in the liver of mice on the LPD (Figure 1B). This increase correlated positively with serum levels. In contrast, Fgf21 expression was unchanged in BAT, eWAT and scWAT of control mice $\left(\right.$ Fgf2 $\left.1^{\text {loxP }}\right)$ on the same diet (Figure $1 C$ ). To determine the specific role of hepatic FGF21 in the metabolic response to a LPD, we fed LFgf21KO mice a LPD diet. As expected, Fgf21 mRNA levels were undetectable in the livers of these animals (Figure 1B), while no statistically significant 
changes were detected in BAT, eWAT or scWAT when compared to the same tissues in control mice (Figure 1C).

This mouse model shows that protein restriction almost exclusively affects the hepatic expression of FGF21 and that there is no compensatory response in other tissues, such as BAT or WAT.

\section{A LPD increases ATF4 protein levels in mouse liver.}

GCN2 is a kinase that acts as a sensor of amino acid supply [28]. When activated, GCN2 phosphorylates EIF2a, which results in the slowing or stalling of the initiation step of mRNA translation. Hence, phospho-EIF2a reduces general protein synthesis rates. Paradoxically, in these circumstances there is an increase in the translation of discrete mRNAs, including ATF4 [29,30]. A LPD increases GCN2-dependent phosphorylation of elF2a, resulting in greater levels of ATF4 protein [31,32]. ATF4 is a transcriptional factor that directly or indirectly induces a subset of specific genes to regulate metabolic adaptation to amino acid restriction. The 5' region of Fgf21 contains two evolutionarily conserved functional ATF4-binding sequences responsible for its ATF4-dependent transcriptional activation $[16,33]$. To determine the effect of a LPD on ATF4 expression, we analyzed liver protein extracts of mice fed a LPD or a CD for 7 days. ATF4 expression was induced in liver in response to the LPD, as revealed by Western blot assays (Figure 2A-B). These results are consistent with previous published data reporting that ATF4 triggers the expression of FGF21 and that Gcn2 -/mice show a partially blunted induction of FGF21 under protein restriction [24]. On the basis of the aforementioned published data, our results support the notion that the GCN2-ATF4 pathway is likely to be the main mechanism underlying hepatic FGF21 overexpression upon protein restriction.

GCN2-independent mechanisms that induce hepatic FGF21 in response to methionine-restricted diets have recently been described [34]. This observation points to a different response program in liver via a non-canonical PERK/nuclear respiratory 
factor 2 (NRF2) pathway. This alternative pathway could effectively sense and translate the metabolic responses to methionine restriction in the absence of GCN2. In parallel, the absence of GCN2 during long-term dietary protein restriction has been reported to be compensated upstream of ATF4 to induce FGF21 [35]. Globally, the impact of alternative pathways to stimulate FGF21 expression under a LPD, whether they involve ATF4 or not, should be addressed in greater depth.

\section{Fgf21 deficiency significantly attenuates weight loss under a LPD}

Mice fed a LPD presented rapid weight loss. Here we addressed whether this phenomenon is dependent on hepatic FGF21. For this purpose, Fgf $21^{\text {LoxP }}$ mice and LFgf21KO mice were fed a CD or LPD for 7 days.

Our data showed that weight loss was partially blunted in LFgf21KO mice (Figure 3AB). However, the reduction in food intake observed under a LPD was unchanged between genotypes (Figure $3 \mathrm{C}$ ). It is remarkable that these results contradicted previous publications describing either no change or an increase in food intake in response to protein restriction [24]. Nonetheless, the present results are consistent with the decreased food intake described in mice fed leucine-deficient diets [19]. Minor changes in diet composition affecting amino acid bioavailability may explain these discrepancies. Moreover, although FGF21 has the potential to modulate food preferences $[36,37]$. In our mouse model this FGF21 did not contribute to the food aversion caused by the LPD.

To determine the importance of each tissue in overall weight loss, we calculated the change in weight of individual tissues. All tissues analyzed tended to weigh less in mice on the LPD, reaching statistical significance in heart, liver, scWAT and $p=0.06$ in eWAT (Figure 4). Regarding the role of FGF21, our results show that the weight loss observed in scWAT and heart was dependent on hepatic FGF21 expression, as weight loss was blunted in LFgf21KO mice under the same diet. The effect of the LPD on liver tissue weight was partially abolished by hepatic Fgf21 deficiency (Figure 4). 
Taken together, FGF21 produced by the liver is, at least in part, responsible for the body weight loss experienced by the mice on the LPD. Our results point to scWAT as one of the target tissues of hepatic FGF21 regarding the weight loss effect. Since hepatic FGF21 exerts its effects mainly in WAT and BAT through regulating lipid metabolism, the following experiments are focused on describing the role of LPDinduced FGF21 on the metabolic response of adipose depots.

\section{LPD induces metabolic changes in response to FGF21 in scWAT, but not in EWAT or BAT.}

Thermogenesis in BAT is mediated by the upregulation of UCP1 [38]. It has been proposed that the induction of FGF21 production by the liver mediates direct activation of brown fat thermogenesis during the fetal-to-neonatal transition [39]. FGF21 also regulates peroxisome proliferator-activated receptor gamma coactivator 1-alpha (PGC1a) and browning of WAT in adaptive thermogenesis [40].

Contrary to what happens under leucine deprivation [19], no statistically significant induction of Ucp1 or Dio2 mRNA levels were observed in BAT or eWAT of control mice under the LPD (Figure 5A-B). In contrast, the analysis of gene expression in scWAT revealed that the LPD induced the expression of Ucp1, Pgc1a, Cidea and PR domain containing 16 (Prdm16), reaching a statistically significant value for Ucp1 and Pgc1a (Figure 6). This expression pattern was not detected in the LFgf21KO mice (Figure 6), thereby indicating the role of FGF21 in the metabolic adaptation of scWAT to protein restriction

As UCP1 activity is related to EE, the blunted induction of UCP1 in the LFgf21KO mice under LPD may contribute to the lower weight loss observed in this mouse model under these circumstances. In conclusion, hepatic FGF21 induces the browning of scWAT and increases the thermogenic capacity of mice on a LPD.

FGF21 plasma levels correlate negatively with protein intake in humans. 
To translate our results to humans, we evaluated the relationship between protein intake and circulating levels of FGF21 in 78 individuals randomly selected from the PREDIMED trial. Baseline data for these subjects are shown in Table 1. Protein intake was obtained from FFQs and was expressed as grams of protein per day (g/day). We used baseline samples $(T=0)$. Results from the multiple linear regression analyses showed a significant inverse relationship between plasma FGF21 concentrations and dietary intake of protein. At baseline, FGF21 levels decreased by $3.39 \mathrm{pg} / \mathrm{mL}$ for each gram of protein ingested (Table 2). The participants with a high intake of protein showed statistically significant lower values of circulating FGF21.

We also performed regression analyses using quartiles of protein intake and obtained similar results. After adjustment for BMI and total energy intake, FGF21 decreased (30.7 pg/mL) when moving from the lower to higher quartiles $(p=0.015)$ (Figure 7).

Similarly to the data from mice, these results indicate that the serum concentrations of FGF21 are inversely proportional to dietary protein intake.

\section{CONCLUDING REMARKS}

Here we addressed the role of hepatic FGF21 in the metabolic changes triggered by a LPD. Our results demonstrate that the effects of a LPD depend, at least in part, on the circulating levels of FGF21 and consequently on the liver production of this growth factor. The LFgf21KO mice revealed the relevance of FGF21 in the response to a LPD, but also in the metabolic and transcriptional pathways activated or repressed by protein restriction.

Given the consistency between the results of our study in humans and those in mice, we postulate that dietary protein content is crucial for the modulation of circulating FGF21 levels and thus for the activity of this hormone in target tissues. We propose a dietary intervention consisting of a reduction in protein intake as a non-invasive approach to induce the hepatic expression of FGF21. We also describe the molecular 
mechanisms through which a LPD—via FGF21—could be beneficial to restore lipid/glucose homeostasis.

Studies performed in humans provide contradictory results regarding the correlation between plasma levels of FGF21, BMI, and insulin resistance [41-43]. Also, the FGF21-resistant state described in mice [44] is not well established in humans and the beneficial effects of FGF21 induction have yet to be demonstrated in the latter.

Our findings provide new insight into the modulation of dietary protein as a strategy to induce elevated serum concentrations of FGF21. Further studies will be needed to evaluate the effects of a LPD / FGF21 induction on the metabolic profile of obese and insulin-resistant subjects.

\section{FOOTNOTES}

Author's contribution: APM performed the experiments with mice. MGG perform the ELISA and the statistical analysis of human samples. ATR performed the statistical analysis of human samples with MGG. RE, JSS, MAMG provided the human samples PFM, DH, JR conceived the project and wrote the paper. All authors read, approved and contributed with important intellectual content to the final version of the manuscript.

Acknowledgements: This project was supported by grant SAF2013-41093 (to PFM and DH) from Spain's Ministerio de Economia y Competitividad, and by funding from the Catalan government (Ajut de Suport als Grups de Recerca de Catalunya 2014SGR916). APM was supported by Scholarship from Spain's Ministerio de Educación Cultura y Deporte.

Conflict of interest: The authors declare they have no conflicts of interest in this article 


\section{REFERENCES}

[1] Kharitonenkov, A. and Shanafelt, A.-B., FGF21: a novel prospect for the treatment of metabolic diseases. Curr. Opin. Investig. Drugs. 2009, 10 (4), 359364.

[2] Fisher, F.-M., Kleiner, S., Douris, N., Fox, E.-C., et al., FGF21 regulates PGC-1a and browning of white adipose tissues in adaptive thermogenesis. Genes Dev. 2012, 26(3), 271-281.

[3] Lee, P., Swarbrick, M.-M. and Greenfield, J.-R., The sum of all browning in FGF21 therapeutics. Cell Metab. 2015, 21(6), 795-796.

[4] Véniant, M.-M., Sivits, G., Helmering, J., Komorowski, R., et al., Pharmacologic Effects of FGF21 Are Independent of the 'Browning' of White Adipose Tissue. Cell Metab. 2015, 21(5), 731-738.

[5] Samms, R.-J., Smith, D.-P., Cheng, C.-C., Antonellis, P.-P., et al., Discrete Aspects of FGF21 In Vivo Pharmacology Do Not Require UCP1.Cell Rep. 2015, 11(7), 991-999.

[6] Nishimura, T., Nakatake, Y., Konishi, M., and Itoh N., Identification of a novel FGF, FGF-21, preferentially expressed in the liver. Biochim. Biophys. Acta Gene Struct. Expr. 2000, 1492(1), 203-206.

[7] Johnson, C.-L., Weston, J.-Y., Chadi, S.-A., Fazio, E.-N., et al., Fibroblast Growth Factor 21 Reduces the Severity of Cerulein-Induced Pancreatitis in Mice. Gastroenterology. 2009, 137(5),1795-1804.

[8] Izumiya, Y., Bina, H.-A., Ouchi, N., Akasaki, Y., et al., FGF21 is an Akt-regulated myokine. FEBS Lett. 2008, 582(27), 3805-3810.

[9] Hondares, E., Iglesias, R., Giralt, A., Gonzalez, F.-J., et al., Thermogenic activation induces FGF21 expression and release in brown adipose tissue. J. Biol. Chem. 2011, 286(15), 12983-12990.

[10] Badman, M.-K., Pissios, P., Kennedy, A.-R., Koukos, G., Hepatic Fibroblast Growth Factor 21 Is Regulated by PPARa and Is a Key Mediator of Hepatic Lipid 
Metabolism in Ketotic States. Cell Metab. 2007, 5(6), 426-437.

[11] Inagaki, T., Dutchak, P., Zhao, G., Ding, X., et al., Endocrine Regulation of the Fasting Response by PPARa-Mediated Induction of Fibroblast Growth Factor 21. Cell Metab. 2007, 5(6), 415-425.

[12] Gälman, C., Lundåsen, T., Kharitonenkov, A., Bina, H.-A., et al,. The Circulating Metabolic Regulator FGF21 Is Induced by Prolonged Fasting and PPARa Activation in Man. Cell Metab. 2008, 8(2), 169-174.

[13] Lundåsen, T., Hunt, M.-C., Nilsson, L.-M., Sanyal, S., et al., PPARa is a key regulator of hepatic FGF21. Biochem. Biophys. Res. Commun. 2007, 360(2), 437-440.

[14] Reitman, M.-L., FGF21: A Missing Link in the Biology of Fasting. Cell Metabolism. 2007, 5(6), 405-407.

[15] Chartoumpekis, D.-V., Habeos, I.-G., Ziros, P.-G., Psyrogiannis, A.-I., et al., Brown adipose tissue responds to cold and adrenergic stimulation by induction of FGF21. Mol. Med., 2011, 17(7-8), 736-740.

[16] De Sousa $\square$ Coelho, A.-L., Marrero, P.-F. and Haro, D., Activating transcription factor 4-dependent induction of FGF21 during amino acid deprivation. Biochem. J., 2012, 443(1), 165-171.

[17] Kim, K.-H., Jeong, Y.-T., Oh, H., Kim, S.-H., et al., Autophagy deficiency leads to protection from obesity and insulin resistance by inducing Fgf21 as a mitokine. Nat Med. 2013, 19(1), 83-92.

[18] Cheng, Y., Meng, Q., Wang, C., Li, H., et al., Leucine deprivation decreases fat mass by stimulation of lipolysis in white adipose tissue and upregulation of uncoupling protein 1 (UCP1) in brown adipose tissue. Diabetes, 2010, 59(1), $17-25$.

[19] De Sousa-Coelho, A.-L., Relat, J., Hondares, E., Pérez-Martí, A., et al., FGF21 mediates the lipid metabolism response to amino acid starvation. J. Lipid Res. 2013, 54(7), 1786-1797. 
[20] Ables, G.-P., Perrone, C.-E., Orentreich, D., and Orentreich, N., MethionineRestricted C57BL/6J Mice Are Resistant to Diet-Induced Obesity and Insulin Resistance but Have Low Bone Density. PLoS One. 2012, 7(12).

[21] Stone, K.-P., Wanders, D., Orgeron, M., Cortez, C.-C. and Gettys, T.-W., Mechanisms of Increased in Vivo Insulin Sensitivity by Dietary Methionine Restriction in Mice. Diabetes. 2014, 63, 1-28.

[22] Ozaki, Y., Saito, K., Nakazawa, K., Konishi, M., et al., Rapid increase in fibroblast growth factor 21 in protein malnutrition and its impact on growth and lipid metabolism. Br. J. Nutr. 2015, 114(9), 1410-1418.

[23] Pezeshki, A., Zapata, R.-C., Singh, A., Yee, N.-J., and Chelikani, P.-K., Low protein diets produce divergent effects on energy balance. Sci. Rep. 2016, 6, 25145.

[24] Laeger, T., Henagan, T.-M., Albarado, D.-C., Redman, L.-M., et al., FGF21 is an endocrine signal of protein restriction. J. Clin. Invest. 2014, 124(9), 3913-3922.

[25] Yakar, S., Liu, J.-L., Stannard, B., Butler, A., et al., Normal growth and development in the absence of hepatic insulin-like growth factor I. Proc. NatI. Acad. Sci. 1999, 96(13), 7324-7329.

[26] Estruch, R., Ros, E., Salas-Salvadó, J., Covas, M.-I., et al., Primary prevention of cardiovascular disease with a Mediterranean diet. N. Engl. J. Med. 2013, 368(14), 1279-1290.

[27] Mataix, J., Tabla de composición de alimentos. 2003, 5a ed. Granada: Universidad de Granada.

[28] Qiu, H., Dong, J., Hu, C., Francklyn, C.-S. and Hinnebusch, A.-G., The tRNAbinding moiety in GCN2 contains a dimerization domain that interacts with the kinase domain and is required for tRNA binding and kinase activation. EMBO J., 2001, 20(6), 1425-1438.

[29] Hao, S., Sharp, J.-W., Ross-Inta, C.-M., McDaniel, B.-J., et al., Uncharged tRNA and sensing of amino acid deficiency in mammalian piriform cortex. Science. 
2005, 307(5716), 1776-1778.

[30] Shan, J., Ord, D., Ord, T. and Kilberg, M.-S., Elevated ATF4 expression, in the absence of other signals, is sufficient for transcriptional induction via CCAAT enhancer-binding protein-activating transcription factor response elements. J. Biol. Chem. 2009, 284(32), 21241-21248.

[31] Anthony, T.-G., McDaniel, B.-J., Byerley, R.-L., McGrath, B.-C., et al., Preservation of liver protein synthesis during dietary leucine deprivation occurs at the expense of skeletal muscle mass in mice deleted for elF2 kinase GCN2. J. Biol. Chem. 2004, 279(35), 36553-36561.

[32] Guo, F. and Cavener, D.-R., The GCN2 elF2a Kinase Regulates Fatty-Acid Homeostasis in the Liver during Deprivation of an Essential Amino Acid. Cell Metab., 2007, 5(2), 103-114.

[33] Wan, X.-S., Lu, X.-H., Xiao, Y.-C., Lin, Y., et al., ATF4- and CHOP-dependent induction of FGF21 through endoplasmic reticulum stress. Biomed Res. Int., vol. 2014, 2014:807874.

[34] Wanders, D., Stone, K.-P., Forney, L.-A., Cortez, et al., Role of GCN2independent signaling through a non-canonical PERK/NRF2 pathway in the physiological responses to dietary methionine restriction. Diabetes, 2016, 65(February), db151324.

[35] Laeger, T., Albarado, D.-C., Burke, S.-J., Trosclair, L., et al., Metabolic Responses to Dietary Protein Restriction Require an Increase in FGF21 that Is Delayed by the Absence of GCN2. Cell Rep., 2016, 16(3), 707-716.

[36] von Holstein-Rathlou, S., BonDurant, L.-D., Peltekian, L., Naber, M.-C., et al., FGF21 Mediates Endocrine Control of Simple Sugar Intake and Sweet Taste Preference by the Liver. Cell Metab. 2016, 23(2), 335-343.

[37] Chu, A.-Y., Workalemahu, T., Paynter, N.-P., Rose, L.-M., et al., Novel locus including FGF21 is associated with dietary macronutrient intake. Hum. Mol. Genet. 2013, 22(9), 1895-1902. 
[38] Matthias, A., Ohlson, K.-B., Fredriksson, J.-M., Jacobsson, A., et al., Thermogenic responses in brown fat cells are fully UCP1-dependent. UCP2 or UCP3 do not substitute for UCP1 in adrenergically or fatty scid-induced thermogenesis. J. Biol. Chem. 2000, 275(33), 25073-25081.

[39] Hondares, E., Rosell, M., Gonzalez, F.-J., Giralt, M., et al., Hepatic FGF21 expression is induced at birth via PPARalpha in response to milk intake and contributes to thermogenic activation of neonatal brown fat. Cell Metab. 2010, 11(3), 206-212.

[40] Fisher, F.-F., Kleiner, S., Douris, N., Fox, E.-C., et al., FGF21 regulates PGC-1a and browning of white adipose tissues in adaptive thermogenesis. Genes Dev. 2012, 26(3), 271-281.

[41] Zhang, X., Yeung, D.-C.-Y., Karpisek, M., Stejskal, D., et al., Serum FGF21 levels are increased in obesity and are independently associated with the metabolic syndrome in humans. Diabetes. 2008, 57(5), 1246-1253.

[42] Chen, W.-W., Li, L., Yang, G.-Y., Li, K., et al., Circulating FGF-21 levels in normal subjects and in newly diagnose patients with Type 2 diabetes mellitus. Exp. Clin. Endocrinol. Diabetes. 2008, 116(1), 65-68.

[43] Chavez, A.-O., Molina-Carrion, M., Abdul-Ghani, M.-A., Folli, F., et al., Circulating fibroblast growth factor-21 is elevated in impaired glucose tolerance and type 2 diabetes and correlates with muscle and hepatic insulin resistance. Diabetes Care. 2009, 32(8), 1542-1546.

[44] Fisher, F.-M., Chui, P.-C., Antonellis, P.-J., Bina, H.-A., et al., Obesity is a fibroblast growth factor 21 (FGF21)-resistant state. Diabetes. 2010, 59(11), 2781-2789. 
Table 1. Baseline characteristics of participants from the PREDIMED cohort included in this study, divided into quartiles of energy-adjusted protein intake at baseline ${ }^{a}$

\begin{tabular}{|l|l|l|l|l|l|}
\hline & \multicolumn{3}{|l|}{ Quartiles of energy-adjusted protein intake } & \\
\hline & Q1 & Q2 & Q3 & Q4 & P value $^{\text {b }}$ \\
\hline No subjects (78) & 19 & 20 & 20 & 19 & \\
\hline Age (years) & $67.1 \pm 5.9$ & $65.7 \pm 4.8$ & $67.0 \pm 6.4$ & $65.3 \pm 4.0$ & 0.30 \\
\hline Sex (women) & $6(31)$ & $10(50)$ & $9(45)$ & $15(79)$ & 0.03 \\
\hline Body mass index & $28.1 \pm 3.2$ & $30.0 \pm 3.4$ & $27.7 \pm 2.7$ & $30.8 \pm 2.9$ & \multirow{2}{*}{0.006} \\
\hline (Kg/m ${ }^{2}$ ) & & & & & \\
\hline Energy intake (Kcal/d) & $2370 \pm 350$ & $2192 \pm 603$ & $2227 \pm 433$ & $2390 \pm 571$ & 0.51 \\
\hline Protein intake (g/day) & $80 \pm 6$ & $90 \pm 2$ & $97 \pm 3$ & $110 \pm 5$ & $<0.0001$ \\
\hline FGF21 (pg/mL) & $289 \pm 116$ & $276 \pm 143$ & $256 \pm 117$ & $190 \pm 115$ & 0.07 \\
\hline
\end{tabular}

${ }^{a}$ Categorical variables: subjects (percentage), continuous variables: mean $\pm S D$

${ }^{\mathrm{b}}$ One-way ANOVA tests (continuous variables) or chi-squared tests (categorical variables). 
Table 2. Multivariable regression analyses with FGF21 $(\mathrm{pg} / \mathrm{mL})$ as dependent variable and energy-adjusted protein intake at baseline (g/day) as independent variable.

\begin{tabular}{|l|l|l|l|l|}
\hline & & $\boldsymbol{\beta}^{\mathrm{a}}$ & $\mathbf{P}$ value & $\mathbf{9 5 \%} \mathbf{C l}$ \\
\hline Protein intake (continuous variable) & Model $1^{\mathrm{b}}$ & -3.42 & 0.006 & $-5.83,-1.02$ \\
\cline { 2 - 5 } & Model $2^{\mathrm{c}}$ & -3.39 & 0.007 & $-5.86,-0.92$ \\
\hline \multirow{2}{*}{ Quartiles of protein intake } & Model $1^{\mathrm{b}}$ & -31.5 & 0.01 & $-56.5,-6.5$ \\
\cline { 2 - 5 } & Model $2^{\mathrm{C}}$ & -30.8 & 0.02 & $-56.5,-5.0$ \\
\hline
\end{tabular}

Cl: Confidence Interval.

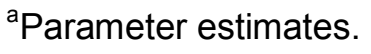

${ }^{\mathrm{b} U n a d j u s t e d .}$

${ }^{\mathrm{c}}$ Adjusted for body mass index (BMI) and total energy intake. 


\section{B}
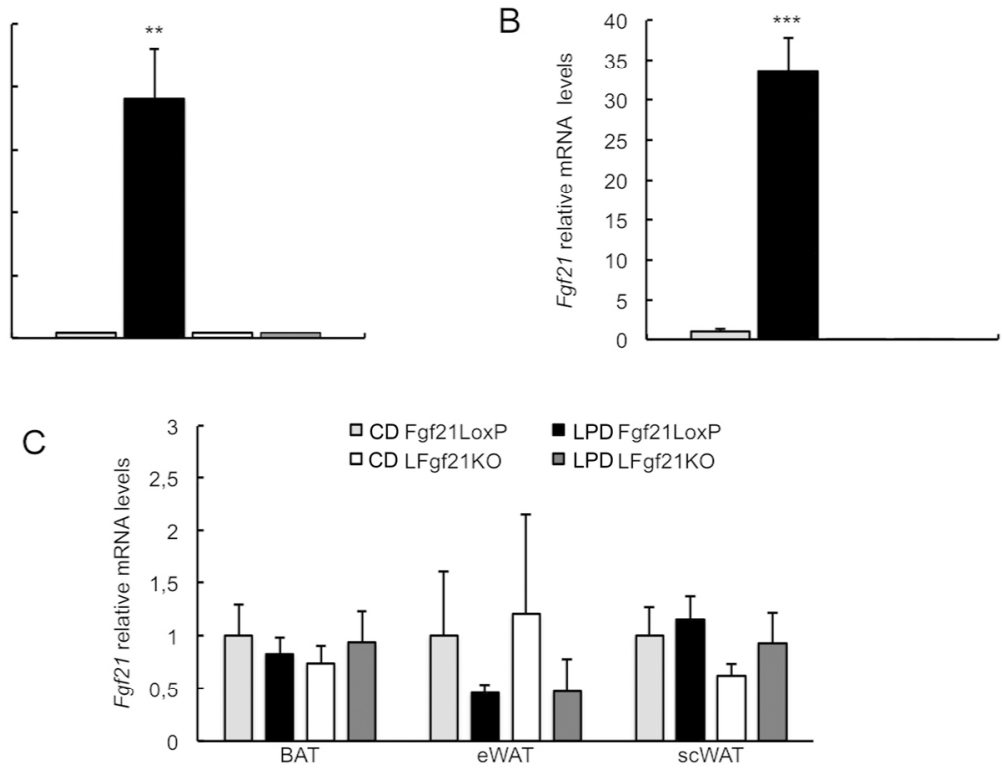

Figure 1. FGF21 is induced by a LPD in liver but not in BAT or WAT, and this induction correlates positively with plasma concentration in mice. Plasma protein concentration of FGF21 was measured by ELISA (A). Fgf21 mRNA levels in liver (B) and WAT and BAT (C) were measured by qRT-PCR. Error bars represent the mean \pm SEM. ** $\mathrm{p}<0.01 * * * \mathrm{p}<0.001$ versus Fgf2 $1^{\text {LoxP }}$ mice fed a $C D(n=7-9 /$ group $)$.

$80 \times 53 \mathrm{~mm}(600 \times 600 \mathrm{DPI})$ 


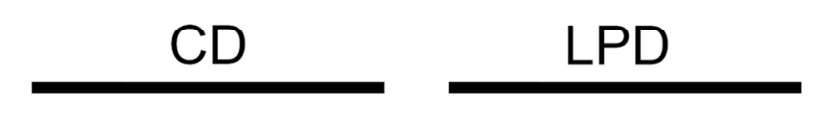

\section{ATF4}

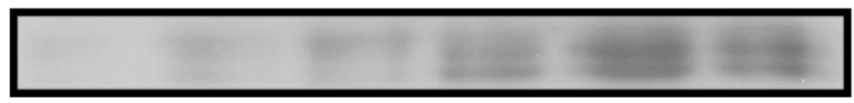

\section{$\beta-A C T I N$}

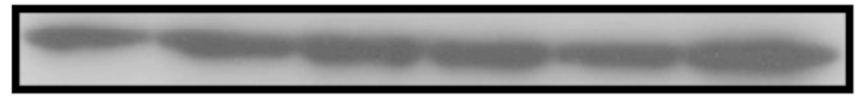

B

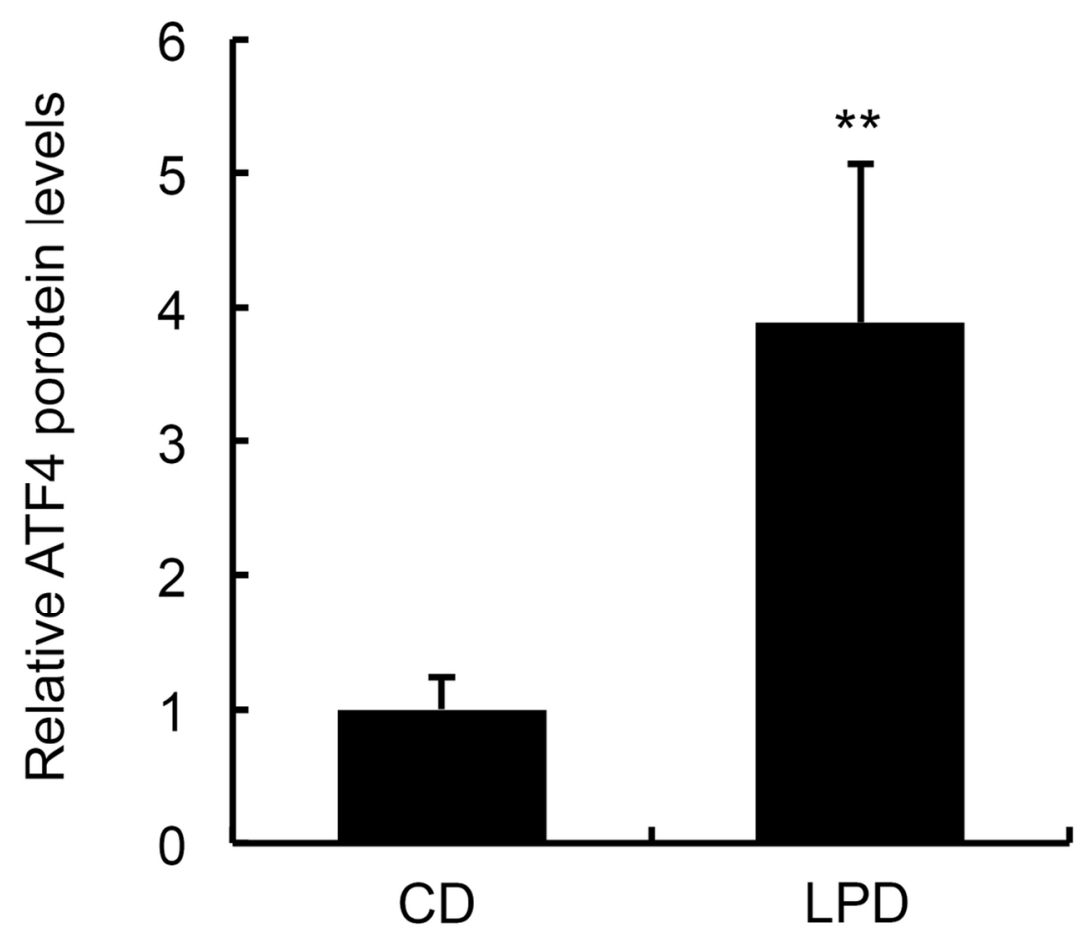

Figure 2. A LPD increases ATF4 protein levels in liver. ATF4 protein levels were determined by Western blot analysis using hepatic nuclear extracts obtained from Fgf $21^{\text {LoxP }}$ mice administered a CD or LPD (A). The experiment was normalized by actin protein levels as loading control and the intensity of the bands were quantified by densitometry with the Image J software (B). Error bars represent the mean \pm SEM. $* * p<0.01$ versus $C D$. ( $n=3 /$ group).

$59 \times 72 \mathrm{~mm}(600 \times 600$ DPI $)$ 
1

2

3

4

5

6

7

8

9

10

11

12

13

14

15

16

17

18

19

20

21

22

23

24

25

26

27

28

29

30

31

32

33

34

35

36

37

38

39

40

41

42

43

44

45

46

47

48

49

50

51

52

53

54

55

56

57

58

59

60
A

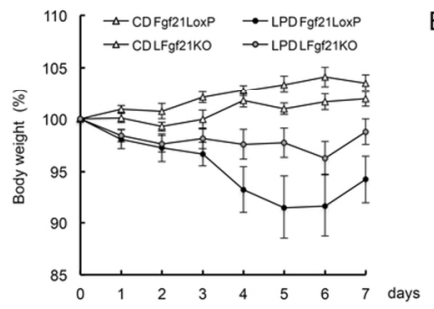

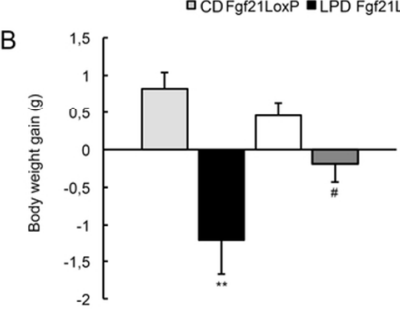

Figure 3. Hepatic FGF21 expression is required for the weight loss caused by LPD but does not affect food consumption. Body weight progression of mice fed a CD or LPD expressed as percentage of the initial weight, which was considered $100 \%(A)$. Total body weight change $(\mathrm{g})$ after 7 days on a CD or LPD (B). Daily food intake (C). Error bars represent the mean \pm SEM. $* * p<0.01$ versus $F g f 21^{\text {LoxP }}$ mice fed a $C D$, \# $p<0.05$ versus LFgf21KO fed a CD; 0.07 represents $p$ value versus LFgf21KO mice fed a $C D(n=7-$ 9/group).

$49 \times 14 \mathrm{~mm}(600 \times 600 \mathrm{DPI})$ 
Figure 4. Hepatic FGF21 is required for the weight loss caused by a LPD. The weight of heart, liver, eWAT, scWAT, BAT and gastrocnemius in mice fed a CD or LPD is presented as the mg of tissue per 100 mg of total body weight. Error bars represent the mean \pm SEM. $* p<0.05 * * * p<0.001$ versus Fgf $21^{\text {LoxP }}$ mice fed a $C D ; \# p<0.05 \# \# p<0.01$ versus $L F g f 21 \mathrm{KO}$ mice fed a CD; 0.06 represents the $\mathrm{p}$ value with respect to Fgf $21^{\text {LoXP }}$ mice fed a CD ( $\mathrm{n}=7-9 /$ group).

$80 \times 53 \mathrm{~mm}(600 \times 600$ DPI $)$ 


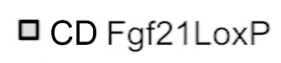

BAT

A

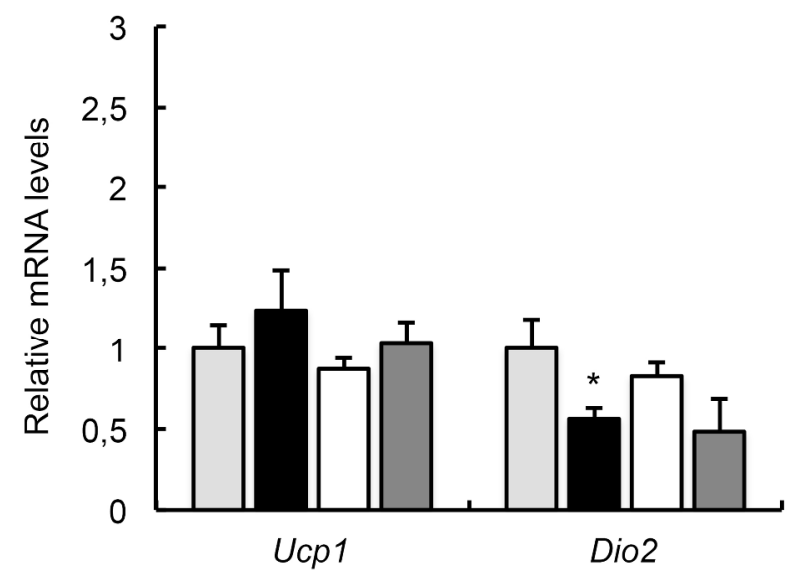

B

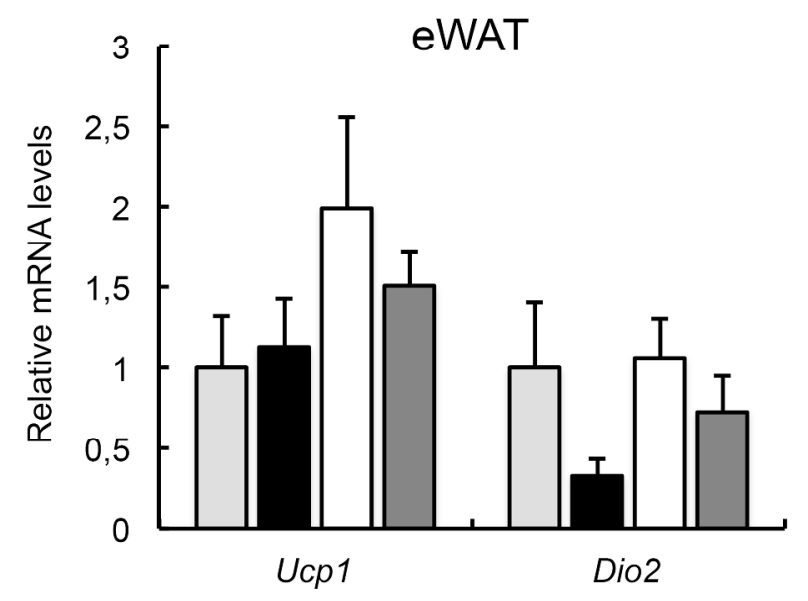

Figure 5. A LPD does not alter thermogenic genes in BAT or eWAT. Ucp1 and Dio2 expression was measured by qRT-PCR in mouse BAT and eWAT. Error bars represent the mean \pm SEM. $*$ p $<0.05$ versus Fgf $21^{\text {LoXP }}$ mice fed a CD ( $\mathrm{n}=7-9 /$ group).

$90 \times 162 \mathrm{~mm}(600 \times 600 \mathrm{DPI})$ 


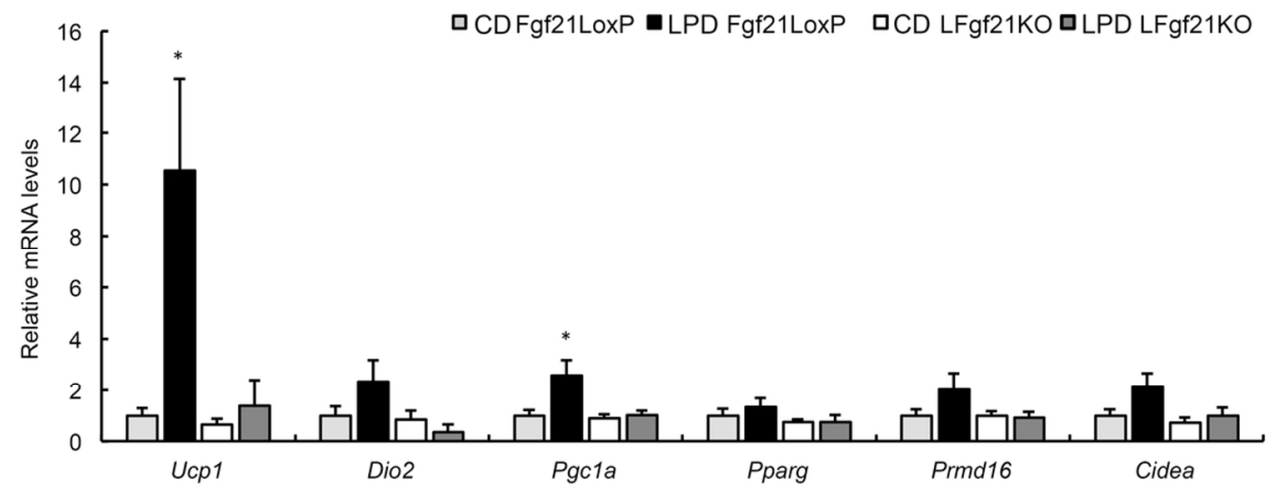

Figure 6. Hepatic FGF21 is required for inducing thermogenic gene expression during a LPD. UCP1, Dio2, Pgc1a, Pparg, Prmd16 and Cidea expression was measured by qRT-PCR in mouse scWAT. Error bars represent the mean \pm SEM. * $\mathrm{p}<0.05$ versus Fgf $21^{\text {LoxP }}$ mice fed a CD $(n=7-9 /$ group $)$.

$49 \times 20 \mathrm{~mm}(600 \times 600 \mathrm{DPI})$ 
Figure 7. Circulating FGF21 levels correlate negatively with protein intake. Plasma FGF21 concentration divided into quartiles of protein intake adjusted for the calorie intake of 78 participants in the PREDIMED trial. Error bars represent the mean \pm SEM. * $p<0.05$ from 1 st quartile; \# $p<0.05$ from the 2 nd quartile. $\vdash \mathrm{T}$

$59 \times 45 \mathrm{~mm}(600 \times 600 \mathrm{DPI})$ 


\section{Graphical Abstract}
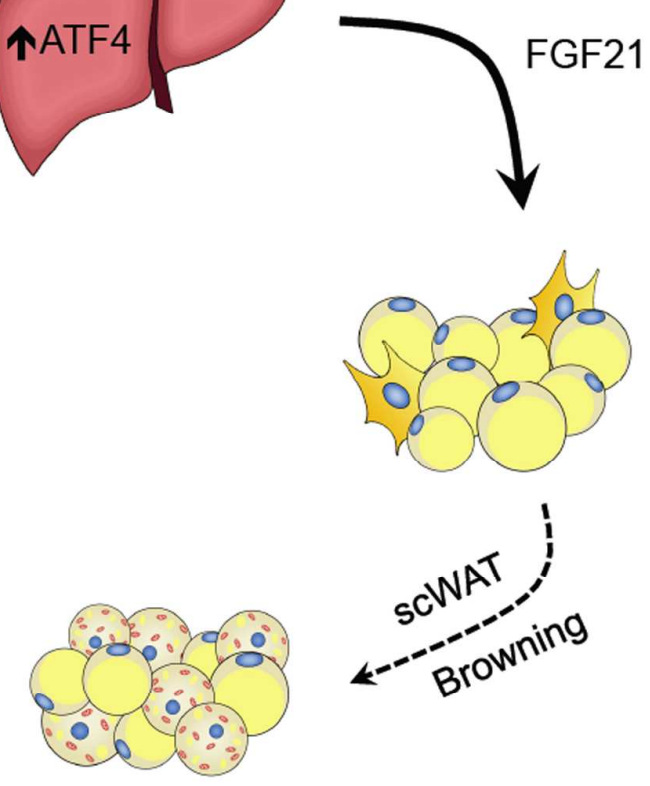

In mice, a low protein diet induces a huge increase in liver FGF21 expression and serum levels, which correlates with enhanced ATF4 protein levels. Also, this diet caused an FGF21-dependent browning of subcutaneous white adipose tissue. The observation of an inverse relationship between serum levels of FGF21 and dietary protein content also in humans suggests that the induction of hepatic FGF21 expression by a low protein diet could offer an effective treatment for obesity and type 2 diabetes. 